\section{Splenic mass in a dog: clinical case report}

\section{ABSTRACT}

Splenic masses, the majority of which are formed by hemangiosarcomas, are frequently observed in dogs of older age compared to other species and ages. A 11-year-old nonneutered male Beagle was admitted to the hospital with the complaints of abdominal distension with non-specific findings such as anorexia, stagnation, and weight loss. Severe abdominal distension with pallor of mucous membranes on clinical examinations; leukocytosis with anemia in the hemogram; high BUN and creatinine levels and a significant increase in liver enzymes in serum biochemistry were determined. In the microscopic examination of the aspirate taken by fine needle aspiration of the splenic mass which determined during ultrasonographic examination, a large number of pleomorphic, multinuclear neoplastic cells with eccentric nuclei, and different amounts of eosinophilic cytoplasm were detected. In this case report, in cases of limitations where biopsy or laparotomy could not be performed due to conditions such as the vascular structure of the mass or patient's disapproval; it was demonstrated that mass presence determined by ultrasonographic examination and the number of infiltrating mast cells determined by microscopic examination of the aspirate taken by fine needle aspiration can provide information in determining the benign or malignant character of the mass.

Keywords: Dog, spleen, neoplasia, hemangiosarcoma, ultrasound, aspiration

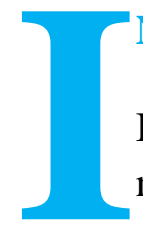

\section{NTRODUCTION}

It has been reported that splenic masses in dogs may be neoplastic such as sarcoma, lymphoma, hemangiosarcoma or non-neoplastic origin such as splenitis, hematoma, lymphoid hyperplastic nodular lesions, and dogs with splenic mass may be asymptomatic as well as with non-specific findings such as lethargy, anorexia, weakness and collapse (Tillson, 2003; Vnuk et al., 2014). Splenic neoplasia is common in elderly and medium to large breed dogs and the hematological and biochemical laboratory findings of are highly variable and non-specific. Although the etiology of the disease is not known exactly, it has been reported that the disorder of the biochemical pathways involved in angiogenesis along with genetic factors plays a role in its development (Thamm, 2007).

In veterinary medicine, abdominal ultrasonography is frequently used in the preoperative evaluation of animals suspected of splenic disease. Splenic masses are often identified by abdominal palpation and/or abdominal ultrasonography/radiography (Tillson, 2003). Although minor changes in splenic composition can be sensitively observed with ultrasonographic imaging, it is not specific for diseases other than splenic abscess or torsion (Vnuk et al., 2014), and the appearance of the spleen differs between diseases (Sato \& Solano, 2004).

\section{How to cite this article}

Gülersoy, E., İyigün, SS., Ertürk, A., Ok, M. (2021). Splenic mass in a dog: clinical case report. Journal of Advances in VetBio Science and Techniques, 6(2), 159-164. https://doi.org/10.31797/vetbio.909520
Research Article

Erdem GÜLERSOY ${ }^{1 a}$ Süleyman Serhat İYIGÜN ${ }^{2 b}$ Alper ERTÜRK ${ }^{2 c}$ Mahmut $\mathrm{OK}^{2 \mathrm{~d}}$

${ }^{1}$ Department of Internal Medicine, Faculty of Veterinary Medicine, Harran University, Şanlıurfa, Turkey

${ }^{2}$ Department of Internal Medicine, Faculty of Veterinary Medicine, Selcuk University, Konya, Turkey

ORCIDa0000-0001-8511-0150 b0000-0002-3270-1931 c0000-0002-0284-2004 d0000-0002-8210-6735

Correspondence Erdem GÜLERSOY egulersoy@yahoo.com Article info

Submission: 06-04-2021

Accepted: 29-06-2021

Online First: 26-08-2021

e-ISSN: 2548-1150 doi prefix: $10.31797 /$ vetbio

- http://dergipark.org.tr/vetbio

This work is licensed under a Creative Commons Attribution 4.0 International License (c) (i) 
Therefore, ultrasound-guided fine needle aspiration and biopsy are used to obtain a diagnosis without the need for invasive surgical interventions (Sabattini \& Bettini, 2009). Cytological or histological evaluation of splenic tissue samples provides information that can eliminate the need for surgical intervention such as benign character or the presence of systemic disease (Ballegeer et al., 2007). When compared to biopsy technique, fine needle aspiration has a lower risk due to needle's fine characteristic that does not cause ruptur or haemorrhage and its features such as not requiring sedation or anesthesia of the animal (Stockhaus \& Teske, 1998).

In this case report, diagnostic methods such as auxiliary hematological and microscopic examinations in the diagnosis of a splenic mass detected in a dog by ultrasonography in conditions of clinical limitations such as lack of biopsy due to vascular structure of the splenic mass or inability to perform laparotomy due to the patient's disapproval are presented.

\section{CASE DESCRIPTION}

The material of this case report consisted of 11year-old non-neutered male Beagle which brought to the Animal Hospital of Selcuk University Faculty of Veterinary Medicine, Department of Internal Medicine, with nonspecific findings such as anorexia, stagnation, weight loss, and abdominal distension. The physical examination of the dog revealed dehydration, pallor of the mucous membranes, low body temperature $\left(36.1{ }^{\circ} \mathrm{C}\right)$ and severe abdominal distension. It was determined that palpable lymph nodes such as mandibular and popliteal lymph nodes were indolent and of normal size on palpation. No abnormal sounds and arrhythmias were detected in lung and heart auscultation.

Table 1. Serum biochemistry findings

\begin{tabular}{|lcclcc|}
\hline Serum Chemistry & Values & Range & Direct Bilirubin (mg/dl) & $\mathbf{0 . 8}$ & $\mathbf{0 - 0 . 3}$ \\
\hline BUN (mg/dl) & 106 & $4.70-7.30$ & Phosphorus (mg/dl) & 15.3 & $1.8-6.4$ \\
\hline Creatinine (mg/dl) & 7.3 & $0.8-1.8$ & Albumin (g/dl) & 2.2 & $2.1-3.9$ \\
\hline AST (U/L) & 165 & $10-80$ & Cholesterol (mg/dl) & 368 & $90-205$ \\
\hline ALT (U/L) & 549 & $10-80$ & Calcium (mg/dl) & 7.4 & $8-10.7$ \\
\hline ALP (U/L) & 1148 & $10-80$ & Triglycerides (mg/dl) & 96 & $10-114$ \\
\hline Amylase (U/L) & 1799 & $500-1800$ & Magnesium (mg/dl) & 3.6 & $1.5-3.5 \mathrm{mg} / \mathrm{dl}$ \\
\hline Glucose (mg/dl) & 131 & $70-150$ & GGT (U/L) & 36 & $1-10$ \\
\hline LDH (U/L) & 201 & $75-490$ & Total Protein (g/dl) & 6.5 & $5.4-7.8$ \\
\hline Total Bilirubin (mg/dl) & 1.6 & $0.1-0.6$ & CPK (U/L) & 152 & $50-450$ \\
\hline
\end{tabular}

BUN: Blood urea nitrogen, AST: aspartate aminotransferase, ALT: alanine transaminase, ALP: alkaline phosphatase, LDH: lactate Dehydrogenase, GGT: gamma-glutamyl transferase, CPK: creatine phosphokinase

Heart (124 bpm) and respiratory rate (36 bpm) were within normal reference limits. For further diagnosis, blood gases (ABL90 Flex Radiometer Automatic Analyzer, Denmark), hemogram (MS4e Melet Schloesing Laboratoires, France) and serum biochemistry (BT 3000 plus Biotecnica Instruments SpA autoanalyzer, Italy) analysis along with abdominal ultrasonography (5-7.5 MHz, Mindray DC-6, China) were performed. Hyperlactataemia in blood gases $(3.5 \mathrm{mmol} / \mathrm{L})$, leukocytosis $\left(26.26 \mathrm{~m} / \mathrm{mm}^{3}\right)$ and anemia $(5.25$ $\mathrm{M} / \mathrm{mm}^{3}$ ) in hemogram, high BUN (106 mg / dl), creatinine $(7.3 \mathrm{mg} / \mathrm{dl})$, AST (165 U/L), ALT (549 U/L), ALP (1148 U/L), total bilirubin (1.6 $\mathrm{mg} / \mathrm{dl})$, phosphorus (15.3 mg/dl), GGT (36 U/L) together with low albumin $(2.2 \mathrm{~g} / \mathrm{dl})$ and calcium $(7.4 \mathrm{mg} / \mathrm{dl})$ levels in serum biochemistry were determined. In the abdominal ultrasonography performed following blood analysis, the head and tail borders of the spleen and the hyperechoic 
capsular area of the spleen could not be visualized in the left hypogastric area, which is the normal anatomical region of the spleen, instead a heterogeneous hypoechoic mass with $9.92 \mathrm{~cm}$ diameter with irregular cavernous vascular structure was detected. During the ultrasonographic examination, no abdominal effusion was detected and it was observed that the mass covered the entire splenic area. Considering the vascular characteristic of the splenic mass, transabdominal fine needle aspiration was preferred because of the risk of secondary rupture associated with biopsy.

Table 2. Blood gases and hemogram findings

\begin{tabular}{|c|c|c|c|c|c|}
\hline Blood Gases & Values & Reference & Hemogram & Values & Reference \\
\hline pH & 7.35 & $7.31-7.42$ & $\mathbf{W B C}\left(\mathrm{m} / \mathrm{mm}^{3}\right)$ & 26.26 & $6-17$ \\
\hline $\mathrm{pCO}_{2}(\mathrm{mmHg})$ & 33.4 & $29-42$ & $\operatorname{Lym}\left(\mathrm{m} / \mathrm{mm}^{3}\right)$ & 6.56 & $0.6-5.1$ \\
\hline $\mathrm{pO}_{2}(\mathrm{mmHg})$ & 44.3 & $85-95$ & $\operatorname{Mon}\left(\mathrm{m} / \mathrm{mm}^{3}\right)$ & 0.91 & $0.1-1.7$ \\
\hline $\mathrm{K}(\mathbf{m m o l} / \mathrm{L})$ & 3.8 & $3.6-5.5$ & Gra $\left(\mathrm{m} / \mathrm{mm}^{3}\right)$ & 18.79 & $3-13.6$ \\
\hline $\mathrm{Na}(\mathrm{mmol} / \mathrm{L})$ & 144 & $139-154$ & $\mathbf{R B C}\left(\mathrm{M} / \mathrm{mm}^{3}\right)$ & 5.25 & $5.5-8.5$ \\
\hline $\mathrm{Ca}(\mathrm{mmol} / \mathrm{L})$ & 0.95 & $2.2-3$ & MCV (fl) & 74.6 & $58-73$ \\
\hline $\mathrm{Cl}(\mathrm{mmol} / \mathrm{L})$ & 95 & $102-120$ & MCH (pg) & 25.6 & $19.5-24.5$ \\
\hline Lactate (mmol/L) & 3.5 & $0-2$ & MCHC (g/dL) & 25 & $28-40$ \\
\hline Base excess (mmol/L) & -5.8 & $-4-4$ & Het $(\%)$ & 39.1 & $35-55$ \\
\hline $\mathrm{HCO}_{3}(\mathrm{mmol} / \mathrm{L})$ & 19.2 & $17-24$ & Hb (g/dL) & 9.8 & $10-18$ \\
\hline
\end{tabular}

pH: Power of hydrogen, pCO2: partial pressure of carbondioxide, pO2: partial pressure of oxygen, K: potassium, Na: sodium, Ca: calcium, Cl: chlorine, HCO3: bicarbonate, WBC: leukocyte, Lym: lymphocyte, Mon: monocyte, Gra: granulocyte, RBC: red blood cells, MCV: mean corpuscular volume, $\mathrm{MCH}$ : mean corpuscular haemoglobin, MCHC: mean corpuscular haemoglobin concentration, Hct: hemotocrit, Hb: haemoglobin

For fine needle aspiration, the dog was held in the lateral position and the sample was taken with a 22 gauge, 1.5 inch needle under ultrasound guidance without applying negative pressure in the syringe (Figure 1).

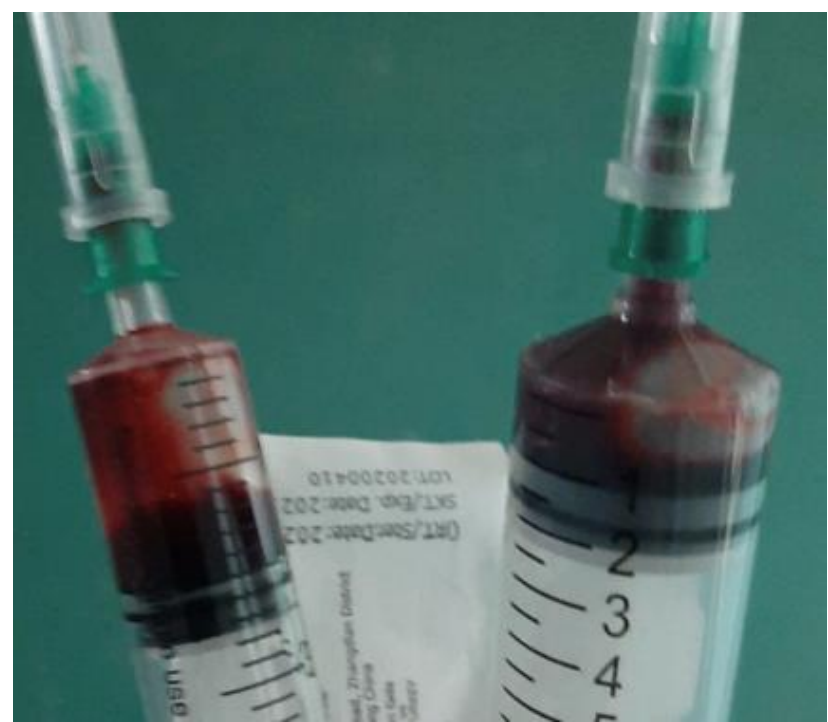

Figure 1. Physical appearance of the aspirate taken from the splenic mass by fine needle aspiration

Wright-Giemsa staining of the aspirate and microscopic examination (Olympus, USA, light microscope x100 magnification) was performed within $1 \mathrm{hr}$ following the procedure. Numerous pleomorphic, multinuclear, neoplastic cells with eccentric nuclei with different amounts of eosinophilic cytoplasm and moderate anisocytosis and anisocaryosis were detected. The dog was humane euthanized with the owner's consent, as the medical condition of the patient did not improve despite palliative treatment.

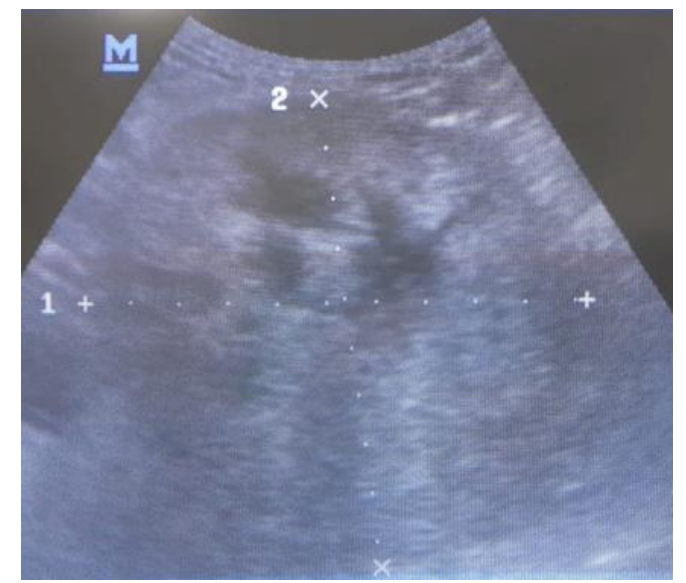

Figure 2. USG image of the splenic mass of $9.92-9.29$ $\mathrm{cm}$ in size (7.5 Mhz). 
Considering the emotional state of the owner, necropsy could not be performed. Since the presence of a splenic neoplastic mass was confirmed in accordance with the anamnesis, physical examination, laboratory and imaging techniques, the necropsy of the dog could not be performed, therefore the benign or malignant character of the mass could not be revealed histopathologically. Serum biochemistry findings in Table 1, blood gases and hemogram findings are presented in Table 2, ultrasonographic image of splenic mass in Figure 2, and multinuclear neoplastic cells determined in microscopic examination are presented in Figure 3.

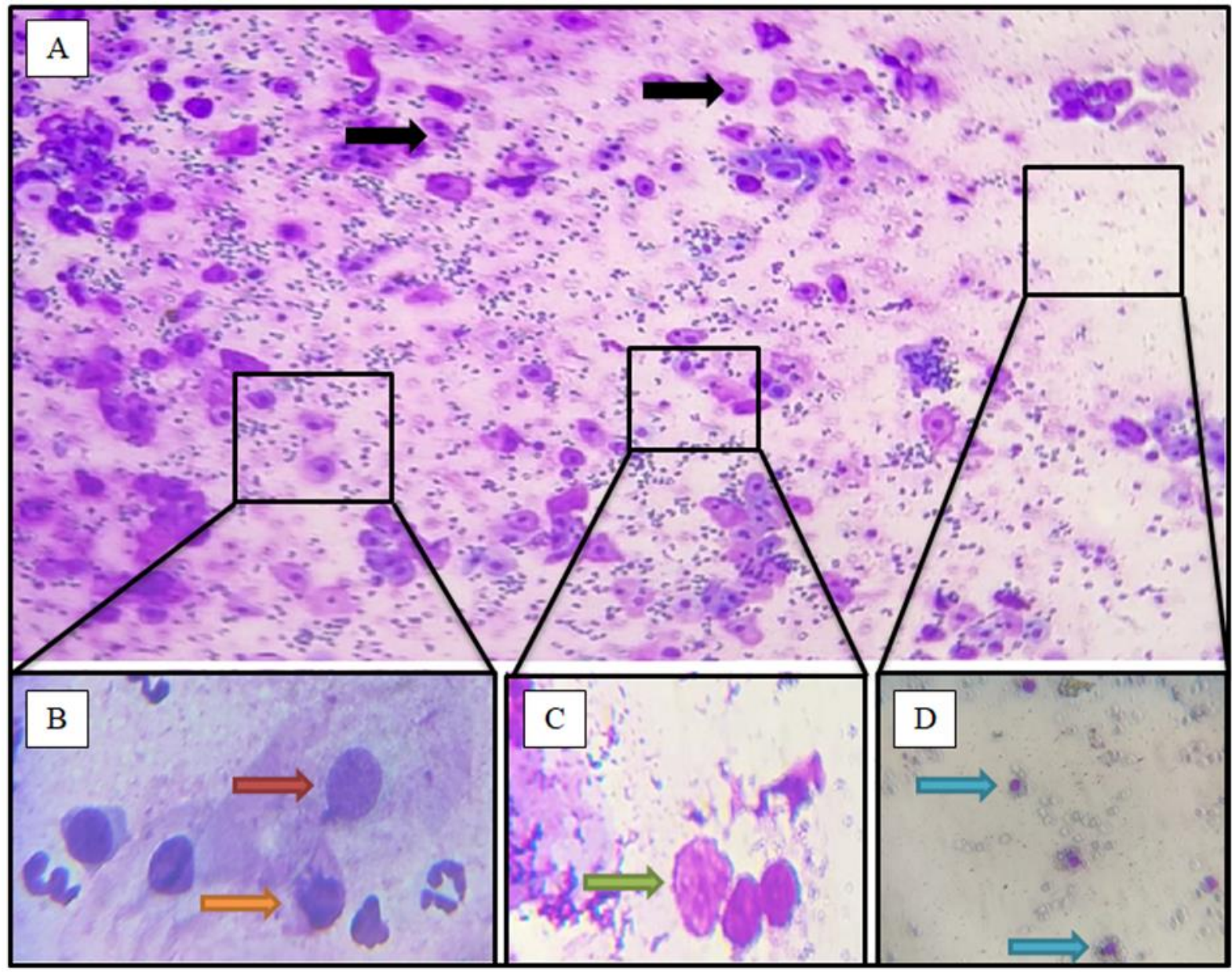

Figure 3. A: Pleomorphic, multinuclear (black arrows) anisocytic neoplastic cells are seen in microscopic examination of the aspirate taken from the spleen by fine needle aspiration, x10 magnification B: Arrows indicate round (red arrow) and eccentric nuclei (orange arrow) of neoplastic cells, x100 magnification with immersion oil C: Neoplastic cells with numerous intracytoplasmic purple granules (green arrow), x100 magnification D: arrows indicate moderate anisocytosis and anisocaryosis (blue arrow), x100 magnification with immersion oil

\section{DISCUSSION}

Spleen; is a hematopoietic organ that has many functions such as blood cell production, haemoglobin iron production, erythrocyte destruction, blood filtration, phagocytosis and immune response (Valli, 2007). In addition to conditions such as trauma and torsion, degenerative, inflammatory, hyperplastic and neoplastic diseases of the spleen can be observed (Fry \& McGavin, 2012). With appropriate anamnesis and blood analysis including hemogram and serum biochemistry, ultrasonographic imaging and ultrasoundguided biopsy or fine needle aspiration provide important clinical information in the diagnosis of splenic disorders in dogs (Macwilliams, 2008). In the hematochemical analysis of dogs with splenic neoplasia, mild to moderate anemia, leukocytosis, and high ALP levels were 
reported (Tillson, 2003). Studies have reported that in hematochemical analysis; regenerative anemia, high ALP, ALT and GGT levels along with weakness, anorexia and abdominal distension in the clinical examination are the most common findings of dogs with splenic mass (Fossum, 2007; Thamm, 2007). In dogs with splenic hemangiosarcoma, increased levels of kidney-related parameters such as BUN, creatinine and liver-related enzymes such as ALP, ALT, AST and GGT have been reported to be associated with tissue hypoxia, hepatic circulatory disorders, cholestasis, dehydration and hemolysis (Gavazza, 2009). Being the mean cell volume $\mathrm{MCV}$, is an important indicator along with $\mathrm{MCH}$ and $\mathrm{MCHC}$ in anemia cases. If reticulocytes are present, the MCV should increase and MCHC decrease, as reticulocytes are in general larger than the RBCs. It is reported that an increased MCHC is always and artifact and a lower MCHC indicate macrocytic hypochromic regenerative anemia as immature RBCs have less haemoglobin (Gröndahl, 2019). Thus, the low RBC and high MCV together with low MCHC levels detected in the hemogram analysis of the present case indicate that anemia was macrocytic hypochromic regenerative characteristics; elevated BUN and creatinine levels due to dehydration and progressive renal function impairement (Adamidis et al., 2010), elevated levels of liver enzymes in the serum biochemistry due to hepatic circulatory impairment; and that hyperlactatemia is due to tissue hypoxia that develops as a result of decreased tissue oxygenation due to anemia. These findings are consistent with previous reported data in dogs with splenic mass/hemangiosarcoma (Smith, 2003; Tillson, 2003).

In a study, it has been reported that the number of infiltrating mast cells is different between malignant and benign vascular tumors of the spleen, and their number is higher in hemangioma cases compared to hemangiosarcoma cases (Sabattini \& Bettini, 2009). In the present case, a large number of pleomorphic, multinuclear, neoplastic cells with eccentric nuclei were detected in the microscopic examination of the aspirate taken from the spleen by fine needle aspiration, but no mast cells were observed. This finding indicates that the mass determined in the present case is neoplastic and may have hemangiosarcoma character due to absence of mast cells (Rodriguez et al., 2020), due to the vascular structure of the mass, lack of biopsy sampling due to the risk of rupture, in cases where laparotomy or necropsy cannot be performed due to the owner's refusal, it shows that the aspirate examination taken with fine needle aspiration can provide important information about the character of the splenic mass (Thamm, 2007; Vnuk et al., 2014).

\section{CONCLUSION}

Diagnostic methods such as fine needle aspiration and magnetic resonance imaging are useful for more accurate diagnosis prior to surgical intervention and histopathological examination. In addition to fine needle aspiration technique, which is effective in the early diagnosis of malignant splenic masses, the owner's acceptance of possible high treatment costs, risks and possible poor prognosis are important factors affecting the survival of the animal.

In conclusion, splenic masses, which are observed more frequently in elderly and medium to large breed dogs, in conditions of clinical limitations such as rupture risk, patient's refusal to take biopsy sample or laparoscopy, abdominal distension in physical examination; macrocytic hypochromic regenerative anemia and leukocytosis in the hemogram; high levels of ALP, ALT and GGT in serum biochemistry; mass presence by ultrasonographic imaging; and the number of infiltrating mast cells in the microscopic examination of the aspirate taken with fine needle aspiration could provide 
information about the benign or malignant character of the mass.

\section{ACKNOWLEDGMENT}

Ethical approval: A dog brought to the Animal Hospital of the Faculty of Veterinary Medicine for diagnosis and treatment constituted the material of the case report. Informed consent was given to the owner of the patient and permission was obtained.

Conflict of interest: The authors state no conflict of interest.

\section{KAYNAKLAR}

Adamidis, K. N., Metaxatos, G., Hadjiconstantinou, V. (2010). Splenic marginal lymphoma and glomerulonephritis: case report and review of the literature. Renal Failure, 32, 281-285.

Ballegeer, E. A., Forrest, L. J., Dickinson, R. M., Schutten, M. M., Delaney, F. A., \& Young, K. M. (2007). Correlation of ultrasonographic appearance of lesions and cytologic and histologic diagnoses in splenic aspirates from dogs and cats: 32 cases (20022005). Journal of the American Veterinary Medical Association, 230(5), 690-696.

Fossum, T. W. (2007). Surgery of the hemolymphatic system. In: Small animal surgery. 3th ed. (pp. 617634). St Louis: Mosby Elsevier, USA.

Fry, M. M., \& McGavin, M. D. (2007). Bone marrow, blood cells, and the lymphatic system. In: Zachary, J. F., McGavin, M. D. (Eds.), Pathologic basis of veterinary disease. 5th ed. (pp. 698-770). St. Louis: Elsevier, USA.

Gavazza, A., Sacchini, F., Lubas, G., Gugliucci, B., \& Valori E. (2009). Clinical, laboratory, diagnostic and prognostic aspects of canine lymphoma: a retrospective study. Comparative Clinical Pathology, 18, 291-299.

Gröndahl, G. (2019). Veterinary Hematology - An introduction. In: Boule Diagnostics. 4th ed. (pp. 153163). Boule Medical, Sweden.

Macwilliams, P. S. (2008). The spleen. In: Cowell, R. L., Tyler, R. D., Meinkoth, J. H., DeNicola, D. B. (Eds.), Diagnostic cytology and hematology of the dog and cat. 3th ed. (pp. 330-337). St. Louis: Mosby Elsevier, USA.

Rodriguez, J. M. M., Morandi, F., Cavicchio, P., Poli, A., \& Verin, R. (2020). Morphological and Immunohistochemical Description of a Splenic Haemangioma in a Captive European Wolf (Canis lupus lupus) and a Review of the Current Literature. Veterinary Sciences, 7(3), 102.

Sabattini, S., \& Bettini, G. (2009). An immunohistochemical analysis of canine haemangioma and haemangiosarcoma. Journal of Comparative Pathology, 140, 158-168.

Sato, A. F., \& Solano, M. (2004). Ultrasonographic findings in abdominal mast cell disease: a retrospective study of 19 patients. Veterinary Radiology and Ultrasound, 45, 51-57.

Smith, A. N. (2003). Hemangiosarcoma in dogs and cats. Veterinary Clinics of North America: Small Animal Practice, 33(3), 533-552.

Stockhaus, C., \& Teske, E. (1998). Clinical experiences with fine needle biopsies of the spleen in diagnosis of canine splenomegaly (in German). Kleintierpraxis, 43, 325-336.

Thamm, D. H. (2007). Miscellaneous tumors: hemangiosarcoma. In: Withrow, S. J. M., Macewen, E. G. (Eds.), Small Anim. Clin. Oncology 4th ed. (pp. 785-795). Philadelphia: WB Saunders Co, USA.

Tillson, D. M. (2003). Spleen. In: SLATTER, D. (Eds.), Textbook of small animal surgery, volume 1, 3rd ed., (pp. 1046-1062). W.B. Saunders, Philadelphia, USA.

Valli, V. E. O. (2007). Hematopoietic system. In: MAXIE, M. G. (Eds.), Jubb, Kennedy and Palmer's pathology of domestic animals. 5 th ed. (pp. 284-290). Philadelphia: Elsevier Saunders, USA.

Vnuk, D., Gusak, V., Schwendenwein, I., Haas, B. M., Musulin, A., \& Maticic, D. (2014). Clinical characteristics and outcomes in 43 dogs with splenic masses of different origin. Veterinary Medicine Austria, 101, 273-280. 